\title{
Topologically Controlled Growth of Magnetic-Metal-Functionalized Semiconductor Oxide Nanorods
}

\author{
Marianna Casavola, ${ }^{\dagger}$ Vincenzo Grillo, ${ }^{\ddagger}$ Elvio Carlino, ${ }^{\ddagger}$ Cinzia Giannini,,$\$$ \\ Fabia Gozzo," Enrique Fernandez Pinel, ${ }^{\perp}$ Miguel Angel Garcia, ${ }^{\perp}$ Liberato Manna, ${ }^{\dagger}$ \\ Roberto Cingolani, ${ }^{\dagger}$ and Pantaleo Davide Cozzoli ${ }^{\star}, \dagger$
}

\begin{abstract}
National Nanotechnology Laboratory of CNR-INFM, Unità di Ricerca IIT, Distretto
Tecnologico ISUFI, via per Arnesano km 5, 73100 Lecce, Italy, TASC-INFM-CNR

National Laboratory, Area Science Park-Basovizza, Bld MM, SS 14, Km 163.5,

34012 Trieste, Italy, CNR-Istituto di Cristallografia (IC), via Amendola 122/O,

70126 Bari, Italy, Swiss Light Source, Paul Scherrer Institute, 5232 Villigen PSI,

Switzerland, and Institute of Applied Magnetism and Department of Materials Physics,

UCM, P.O. Box 155, 28230 Las Rozas, Madrid, Spain
\end{abstract}

Received March 8, 2007; Revised Manuscript Received April 4, 2007

\begin{abstract}
Colloidal semiconductor-magnetic hybrid nanocrystals with topologically controlled composition are fabricated by heterogeneous nucleation of spherical $\epsilon$-Co domains onto anatase $\mathrm{TiO}_{2}$ nanorods. The latter can be selectively decorated at either their tips or at multiple locations along their longitudinal sidewalls, forming lattice-matched heterointerfaces regardless of the metal deposition sites. The possibility of switching between either heterostructure growth modes arises from the facet-dependent chemical reactivity of the oxide seeds, which is governed mainly by selective adhesion of the surfactants rather than by small differences in misfit-induced interfacial strain at the relevant junction points.
\end{abstract}

Over the past decade, considerable progress in many fundamental and practical fields of nanoscience (e.g., in quantum confinement modeling, optoelectronics, magnetic data storage, and biomedicine) has profited from the availability of size- and shape-tailored solution-grown nanocrystals (NCs) with dimensionality-related properties. ${ }^{1}$ A demand for multipurpose materials has now emerged that is orienting efforts of nanochemistry research to envision novel generations of nanoscale solids, able to perform several technological tasks simultaneously. ${ }^{2}$ Only recently, colloidal routes have advanced in this direction, opening access to first prototypes of noncentrosymmetric hybrid nanocrystals (HNCs) with a topologically controlled composition on the sub-100 nm scale. ${ }^{3} \mathrm{HNCs}$ are elaborate noncore/shell heterostructures, which comprise two or more chemically different crystalline sections interconnected through small inorganic interfaces. ${ }^{4-23}$ The permanent grouping of luminescent, magnetic, and

* To whom all correspondence should be addressed. E-mail: davide.cozzoli@unile.it. Telephone: +39 0832 298231. Fax: +390832 298238.

National Nanotechnology Laboratory of CNR-INFM.

$\doteqdot$ TASC-INFM-CNR National Laboratory.

$\S$ CNR-Istituto di Cristallografia.

" Swiss Light Source, Paul Scherrer Institute.

${ }^{\perp}$ Institute of Applied Magnetism and Department of Materials Physics. catalytically active materials in a unique solution-processable particle is expected to pave the way to the creation of "smart" nano-objects with multifunctional capabilities. ${ }^{3}$ Owing to the contact junctions shared by the constituent domains, the physical-chemical behavior of HNCs often deviates from that of the corresponding isolated components, which can be deliberately used to engineer nanostructures with tunable or enhanced performances. ${ }^{9,11,13,17-21}$ Also, HNCs represent potential substrates onto which asymmetric distributions of functional groups can be arranged to mediate bioconjugation, ${ }^{12}$ to facilitate electrical connections, ${ }^{17}$ and to promote nanocrystal self-assembly. ${ }^{24}$

The chemical fabrication of HNCs in liquid media necessarily involves a higher degree of synthetic ingenuity. Indeed, in the formation of a nanoheteroarchitecture, the delicate interplay between thermodynamically and kinetically driven processes (e.g., relative phase stability, surface adhesion of surfactants, monomer diffusion, crystallographic-directiondependent growth rate), which dictate the size and shape of the individual domains, is complicatedly affected by intercompound miscibility, interfacial strain, and facet-specific chemical reactivity. ${ }^{1,3}$ As control over such a complex dynamics is inherently difficult to achieve, the field of HNC 
synthesis is still in its early advancement stages. In fact, just a few examples of HNCs have so far become available, and their growth mechanisms have not been fully elucidated. For example, phase segregation has been exploited to obtain $\mathrm{Co}-$ Pd sulfide ${ }^{4}$ and $\mathrm{Cu}$-In sulfide ${ }^{5}$ heterostructures with a graded interface. Seeded growth techniques have been used to combine magnetic, metallic, and fluorescent particles into heterodimers (such as of $\mathrm{FePt}-\mathrm{CdS},{ }^{6} \gamma$ - $\mathrm{Fe}_{2} \mathrm{O}_{3}$ - metal sulfide, ${ }^{7,8} \mathrm{CoPt}_{3}-\mathrm{Au},{ }^{9} \mathrm{Ag}-\mathrm{Se},{ }^{10} \mathrm{Au}-\mathrm{Fe}_{3} \mathrm{O}_{4},{ }^{11} \mathrm{FePt}-\mathrm{Ag}$, and $\mathrm{Fe}_{3} \mathrm{O}_{4}-\mathrm{Ag}$ ) ${ }^{12}$ and hetero-oligomers (such as of $\mathrm{Au}-\mathrm{Fe}_{3} \mathrm{O}_{4}-$ $\mathrm{Au}, \mathrm{PbS}-\mathrm{Au}-\mathrm{PbS}, \mathrm{Fe}_{3} \mathrm{O}_{4}-\mathrm{Au}-\mathrm{PbSe},{ }^{13}$ and $\mathrm{Pd}-\mathrm{Co}-\mathrm{Pd}$ sulfide). ${ }^{14}$ Facet-preferential heterogeneous nucleation has allowed selected locations of anisotropically shaped NCs to be functionalized with additional materials. Relevant demonstrations include CdS-, CdSe-, and CdTe-based heterostructures with linear or branched topology,${ }_{16}^{15}$ "nanodumbbells" and "nanomatchsticks" of $\mathrm{CdSe}-\mathrm{Au},{ }^{16,17} \mathrm{CdS}-\mathrm{Au},{ }^{18}$ and $\mathrm{CdS}-\mathrm{PbSe},{ }^{19,20} \mathrm{Au}$-edged $\mathrm{PbS}$ nanocubes, ${ }^{21} \mathrm{ZnO}$ and $\mathrm{TiO}_{2}$ nanorods asymmetrically decorated with $\mathrm{Ag},{ }^{22}$ and $\gamma-\mathrm{Fe}_{2} \mathrm{O}_{3}$ spheres, ${ }^{23}$ respectively.

One technologically attractive class of nanocomposites is the association of transition metals with semiconductor oxides. Cooperative electronic interactions between these two materials are responsible for high catalytic activity in a variety of reactions,${ }^{25}$ while enhanced charge carrier separation is manifested by improved photocatalytic and electrochemical responses. ${ }^{26}$ As compared to the successful preparation of oxide-supported metal nanoparticles by exploitation of various chemophysical blending or electrostatic stabilization mechanisms, ${ }^{27}$ the development of topologically defined metal/oxide heterostructures remains mostly limited to onionlike systems ${ }^{26 a, b, d, 28}$ and to a few HNC combinations. ${ }^{11-13,22}$

In this letter, we report on a surfactant-directed approach to synthesize a novel type of semiconductor/magnetic HNC, which comprises metal Co spheres coherently grown on selected locations of one $\mathrm{TiO}_{2}$ rodlike section. The topology of the heterostructures can be straightforwardly controlled by simply adjusting a few synthesis parameters. Our preparation strategy relies on combining two key concepts: first, the use of preformed $\mathrm{TiO}_{2}$ nanorods acting as the nucleation seeds for Co; second, a suitable temporal variation of the surfactant composition in the reaction mixture during the synthesis course. The main steps involved in a typical HNC synthesis are summarized as follows. ${ }^{29}$ Organic-capped anatase $\mathrm{TiO}_{2}$ nanorods (NRs) with different geometric parameters are first prepared by using either hydrolytic or nonhydrolytic modified literature protocols, both of which employ oleic acid (OLAC) as the promoter of anisotropic growth. ${ }^{30}$ Calibrated amounts of thoroughly purified NRs, oleyl amine (OLAM), and 1-octanoic acid (OCAC) in welldefined relative proportions, are co-dissolved in 1-octadecene (ODE) under inert atmosphere and heated up to the temperature selected for the metal precursor decomposition (in the $240-280{ }^{\circ} \mathrm{C}$ range). Metallic cobalt nucleation is induced by the slow addition (controlled by syringe pump) of an oxygen-free $\mathrm{Co}_{2}(\mathrm{CO})_{8} / \mathrm{ODE}$ diluted solution to the vigorously stirred mixture, following which the injection of freshly degassed OLAC is suddenly performed. The formation of

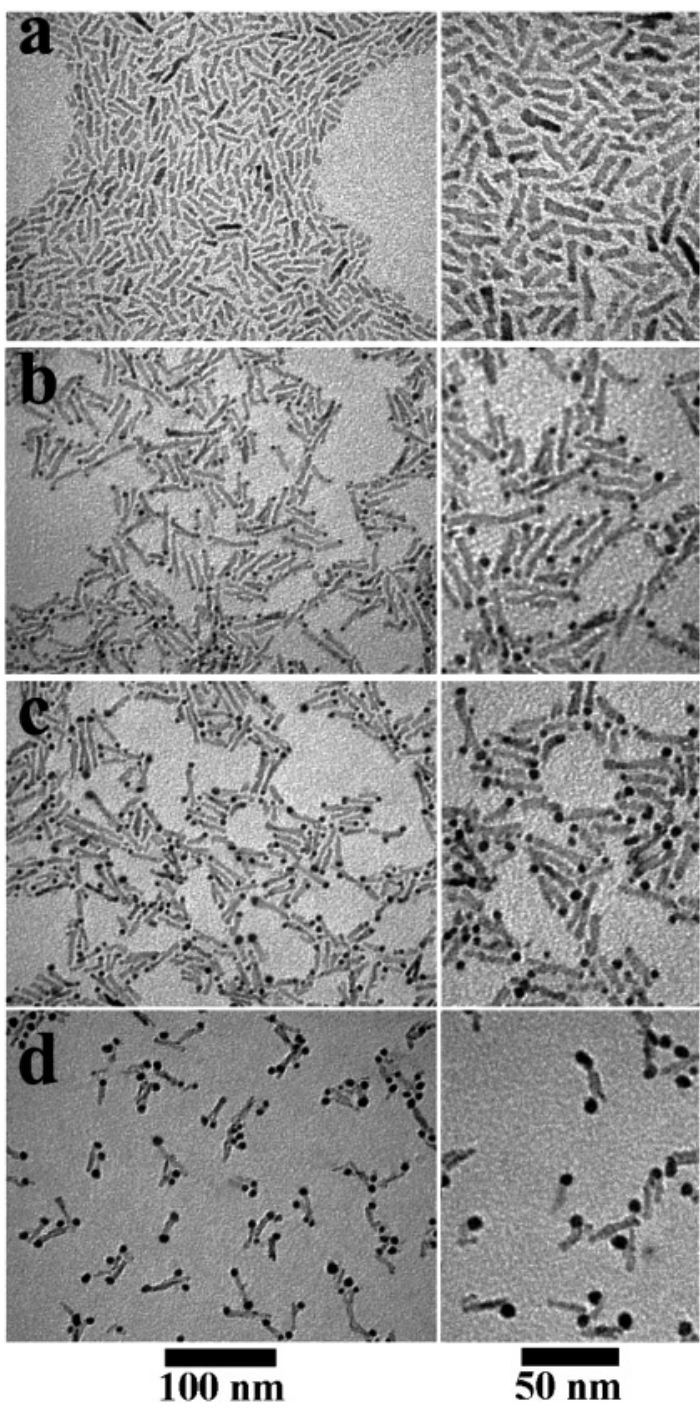

Figure 1. Low-resolution TEM images illustrating the formation of matchstick- and dumbbell-shaped $\mathrm{Co}-\mathrm{TiO}_{2} \mathrm{HNCs}$, synthesized at $250{ }^{\circ} \mathrm{C}$ and at $\mathrm{OCAC} / \mathrm{OLAM} / \mathrm{TiO}_{2}=2: 3: 5$ starting from the hydrolytically prepared $\mathrm{TiO}_{2}$ nanorods. The initial $\left[\mathrm{TiO}_{2}\right]$ was 0.2 $\mathrm{M}$, while the $\mathrm{Co} / \mathrm{TiO}_{2}$ molar ratio was adjusted to: (a) 0 , (b) $1: 1$, (c) $1: 1.5$, (d) $1: 2$, respectively. Typical Co domain size distributions are $15-20 \%$.

heterostructures is achieved by keeping the OCAC/OLAM/ $\mathrm{TiO}_{2}$ molar ratio lower than 3:3:5, while their colloidal stability is guaranteed at OLAC/Co $=3: 1$. After $\mathrm{Co}_{2}(\mathrm{CO})_{8}$ decomposition is allowed to proceed to completion, the reaction is halted by removing the heating source. The final nanocrystalline product, extracted by alcohol addition and purified by repeated washing cycles, is dispersible in a variety of nonpolar solvents (such as hexane, toluene, or chloroform).

The low-magnification transmission electron microscopy (TEM) overview in Figure 1 demonstrates the degree of topological control achievable by seeding the growth of $\mathrm{TiO}_{2}-\mathrm{Co} \mathrm{HNCs}$ with hydrolytically prepared $\mathrm{NRs}^{30 \mathrm{a}}$ under "standard" conditions $\left(T=250{ }^{\circ} \mathrm{C}, \mathrm{OCAC} / \mathrm{OLAM} / \mathrm{TiO}{ }_{2}\right.$ molar ratio is adjusted between 1:3:5 and 3:3:5). ${ }^{29}$ The starting $\mathrm{TiO}_{2}$ NRs (panel a) possess an average length of $\sim 25-30 \mathrm{~nm}$ and a mean diameter of $\sim 3.5-4 \mathrm{~nm}$ and include a $10-15 \%$ fraction of fork-shaped branched objects. Fol- 


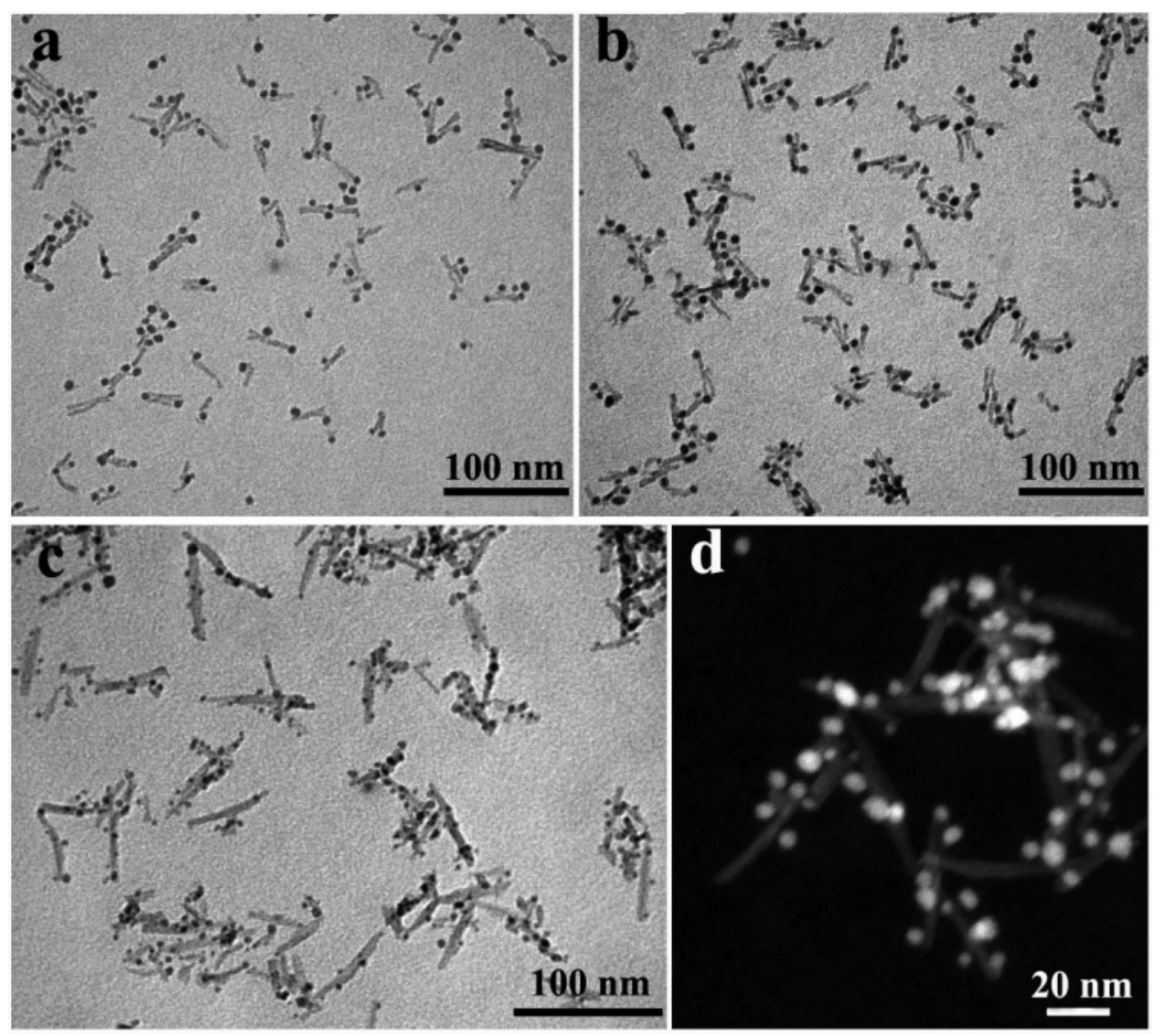

Figure 2. $(\mathrm{a}-\mathrm{c})$ Low-resolution TEM overviews illustrating the formation of $\mathrm{HNCs}$ based on multiply Co-decorated $\mathrm{TiO}_{2}$ nanorods. $(\mathrm{a}-\mathrm{b})$ $\mathrm{HNC}$ growth seeded with hydrolytically synthesized $\mathrm{TiO}_{2}$ nanorods $\left(T=250{ }^{\circ} \mathrm{C},\left[\mathrm{TiO}_{2}\right]=0.2 \mathrm{M}, \mathrm{Co} / \mathrm{TiO}{ }_{2}=1: 1 \mathrm{M}\right)$ and performed at OCAC/OLAM/ $/ \mathrm{TiO}_{2}$ molar ratio of 0.5:3:5 and 0.1:3:5, respectively. (c) HNC growth seeded with nonhydrolytically synthesized larger $\mathrm{TiO}_{2}$ nanorods at $280{ }^{\circ} \mathrm{C}$ (the other conditions were the same as in (a-b). (d) Low-magnification HAADF image of a group of multiply Co-decorated HNCs.

lowing the reaction with $\mathrm{Co}_{2}(\mathrm{CO})_{8}$, the formation of $\mathrm{HNCs}$ is authenticated by the observation of nano-objects with a mixed morphological profile (panels $b-d$ ). Indeed, the newly formed nanostructures appear to be individually composed of an anisotropic rod-shaped section functionalized with either one or two roughly spherical tips, which are characterized by a comparatively higher image contrast. Being aware of the known decomposition paths of $\mathrm{Co}_{2}(\mathrm{CO})_{8}$ in hot surfactant mixtures,$^{31}$ the preliminary TEM inspection suggests that each of the obtained HNCs incorporates one of the initial $\mathrm{TiO}_{2}$ seeds in its elongated portion, while the attached "dark" spherical particles should be made of metallic Co. Such attribution is further supported by our control experiments, which indeed confirmed the structuralmorphological stability of the $\mathrm{TiO}_{2}$ NRs under the typical synthesis conditions. ${ }^{29}$ Notably, the obtained HNCs exhibit a distinct geometric arrangement, as the Co domains are preferentially located in close proximity of the nanorod terminations, which confers either matchstick- or dumbbelllike morphology to the resulting hybrid structures. The mean size of the metal tips can be tuned from $\sim 2$ to $\sim 8 \mathrm{~nm}$ by employing progressively higher $\mathrm{Co}_{2}(\mathrm{CO})_{8} / \mathrm{TiO}_{2}$ molar ratios in the syntheses (panels $\mathrm{b}-\mathrm{d}$ ), beyond which further increases in the precursor to seed proportions ultimately lead to contamination of the HNC samples with homogeneously nucleated Co particles rather than to larger Co tips on the NRs. In all circumstances, the relative abundance of the matchstick- to dumbbell-shaped HNC population (typically $60-70 \%$ to $30-40 \%$ ) remains nearly constant over time.

The formation of permanently fused nanorod-sphere heterojunctions is corroborated by the following observations. First, the relative heterostructure configuration cannot be disrupted after subjecting the samples to repeated cycles of precipitation/redissolution. Second, if required, size-sorting can be carried out by careful nonsolvent-based destabilization of the colloidal solution to enrich the samples with HNCs relative to the other particle types, as the former possess the highest average mass in the population. More interestingly, an external magnetic field can be applied in order to effectively separate the $\mathrm{HNCs}$ from unreacted $\mathrm{TiO}_{2} \mathrm{NRs}^{29}{ }^{29}$

Other types of HNC configurations have been synthesized by carefully adjusting the sole OCAC concentration in the system. Figure 2 demonstrates the possibility of switching from the above-reported tip-preferential metal growth to a nonselective deposition mode, in which Co nucleates also onto the longitudinal sidewalls of the NRs. The comparison between the TEM images in panels $\mathrm{a}-\mathrm{b}$ indicates that the transition to such a topological regime at a given temperature is triggered by progressive reduction in the $\mathrm{OCAC} / \mathrm{TiO}_{2}$ molar ratio below $\sim 1: 5$ (while other reaction parameters are 


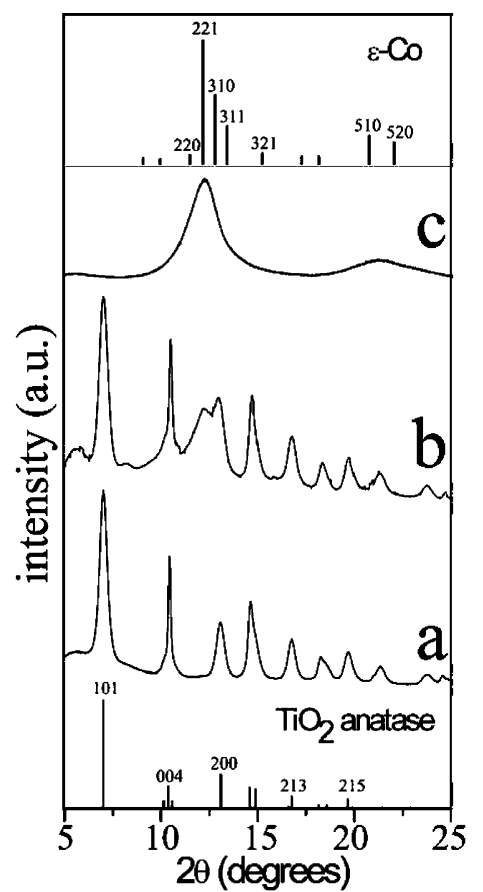

Figure 3. Powder X-ray diffraction patterns of: (a) $3.5 \mathrm{~nm} \times 30$ nm $\mathrm{TiO}_{2}$ nanorods, (b) $\mathrm{TiO}_{2}-\mathrm{Co} \mathrm{HNCs}$ grown with metal domains of $\sim 3.5 \mathrm{~nm}$, (c) $\sim 3.5 \mathrm{~nm}$ Co NCs.

kept constant), upon which both the fraction of NRs with Co-decorated sidewalls and the average number of $\mathrm{Co}$ domains nucleated on a single $\mathrm{TiO}_{2}$ seed are found to increase. Furthermore, panels $\mathrm{c}-\mathrm{d}$ reveal that multiply Codecorated NRs become the dominant product when the reaction is conducted on larger aspect ratio $\mathrm{TiO}_{2} \mathrm{NRs}^{30 \mathrm{~b}}$ and/ or at higher temperatures than those used in the standard conditions. In panel d, the high-angle annular dark-field (HAADF) analysis carried out with a scanning transmission electron (STEM) microscope ${ }^{29}$ confirms the topological variation of the chemical composition of the HNCs, as previously suggested by TEM contrast changes. Indeed, as the HAADF brightness is related to the local sample thickness and to its mean atomic number, it can be inferred that Ti-related species are mainly present in the anisotropically shaped sections of the heterostructures, while Coassociated species dominate in the spherical domains.

The crystal phase composition of the samples has been investigated by powder X-ray diffraction (XRD) analysis performed under inert atmosphere. ${ }^{29}$ Representative results are reported in Figure 3. The patterns generally exhibit significant line broadening, in agreement with the formation of nanosized crystalline domains. The profile of the initial $\mathrm{TiO}_{2}$ rodlike seeds (trace a) is consistent with the standard pattern of the tetragonal anatase $\mathrm{TiO}_{2}$ polymorph, for which the comparatively higher intensity and narrower width of the (004) reflection indicate a preferential crystal elongation along the $c$-axis direction. ${ }^{30}$ In the XRD pattern of the HNC sample (trace b), the characteristic features of the anisotropic $\mathrm{TiO}_{2}$ seeds are still recognizable, while an additional broad diffraction band emerges at around $2 \theta=12^{\circ}$. The latter arises from the convolved contributions of the (220), (221), (310), and (311) reflections of metallic Co in the cubic $\epsilon$-phase $(\epsilon-\mathrm{Co}),{ }^{31}$ which dominate also in the profile of the nanocrystalline cobalt product synthesized in the absence of $\mathrm{TiO}_{2}$ (trace c). As an indirect confirmation for the above attributions, exposure of the $\mathrm{HNCs}$ to ambient atmosphere leads to the detection of a cubic $\mathrm{CoO}$ phase, which results from air-induced oxidation of $\epsilon$-Co. ${ }^{29}$

High-resolution TEM (HRTEM) investigations ${ }^{29}$ provided detailed information on the structure of the HNCs. A few types of $\mathrm{TiO}_{2} / \mathrm{Co}$ heterointerfaces have been found, among which the most frequently observed are described in the following. Figure 4 reports a HRTEM study on a portion of a single matchstick-shaped HNC. The corresponding fast Fourier transform (FFT) analyses in panels $b-c$ indeed demonstrate that the spherical head of the heterostructure is made of $\epsilon$-Co, while the rod-shaped section is $[0,0,1]$ elongated tetragonal anatase $\mathrm{TiO}_{2}$, confirming the XRD results. The two FFT patterns have been merged in panel $d$ to study the relationships between the two lattices. The joint diffractogram indicates that the relative orientation of the interface between the two domains corresponds exactly to the $\{1,3,0\}_{\epsilon-\mathrm{Co}}$ plane and approximately to the $\{0,1,7\}_{\mathrm{TiO} 2}$ plane. A straightforward relationship of planar epitaxy can be identified in the direction orthogonal to the interface, along which the $\{0,3,-3\}_{\mathrm{TiO} 2}$ planes $(d=0.1172 \mathrm{~nm})$ and the $\{-3,1,4\}_{\epsilon-\text { Co }}$ planes $(d=0.1196 \mathrm{~nm})$ (the latter being obtained as a sum of the two $\{1,2,2\}$-like periodicities in panel c) are coincident with a $\sim 2 \%$ misfit. The same condition of epitaxy can be restated in terms of continuity of atomic planes at the junction region. A steady correspondence between the two lattices can be identified, being such that the projection of every three adjacent $\{1,2,2\}_{\epsilon-\mathrm{Co}}$ planes on the observable unidirectional interface coincides regularly with a lattice distance given by the sum of two consecutive $\{1,0,1\}_{\mathrm{TiO} 2}$ spacings. In light of the coincidence site lattice theory, ${ }^{32}$ the involved lattice misfit is $\sim 0.1 \%$. The HRTEM simulation in panel e, where the two domains are positioned as in panel a, and the corresponding FFT pattern in panel $\mathrm{f}$, confirm the above findings. In panel $\mathrm{g}$, an atomic model has been constructed, which represents the HNC in panel e after a $90^{\circ}$ rotation with respect to the interfacial plane. This additional view reveals that the relative $\mathrm{TiO}_{2}-$ Co geometric arrangement attained in the matchstick-like HNC allows the two crystal structures to be coherently connected also in the direction orthogonal to the zone axes that have been captured experimentally. Indeed, under the new orientation, each $\{1,1,1\}_{\epsilon-\text { Co }}$ plane projects, on the visible unidirectional interface, a lattice distance equal to the sum of two consecutive $\{0,2,0\}_{\mathrm{TiO} 2}$ spacings. The corresponding misfit ${ }^{32}$ is almost negligible, as low as $0.26 \%$.

Panels $\mathrm{a}-\mathrm{d}$ in Figure 5 report a HRTEM study of the junction between a $\epsilon$-Co particle grown onto a longitudinal sidewall of a multiply Co-decorated $\mathrm{TiO}_{2}$ nanorod. The FFT analyses show that the interface is parallel to the $\{3,-2,1\}_{\epsilon-\mathrm{Co}}$ and to the $\{0,1,1\}_{\mathrm{TiO} 2}$ planes. For this heterojunction configuration, the epitaxial relationship between the two lattices is established by the regular matching between the projections of the $\{0,0,4\}_{\mathrm{TiO} 2}$ fringes on the interface and the projections of the $\{2,2,1\}_{\epsilon-\mathrm{Co}}$ fringes, respectively, which 

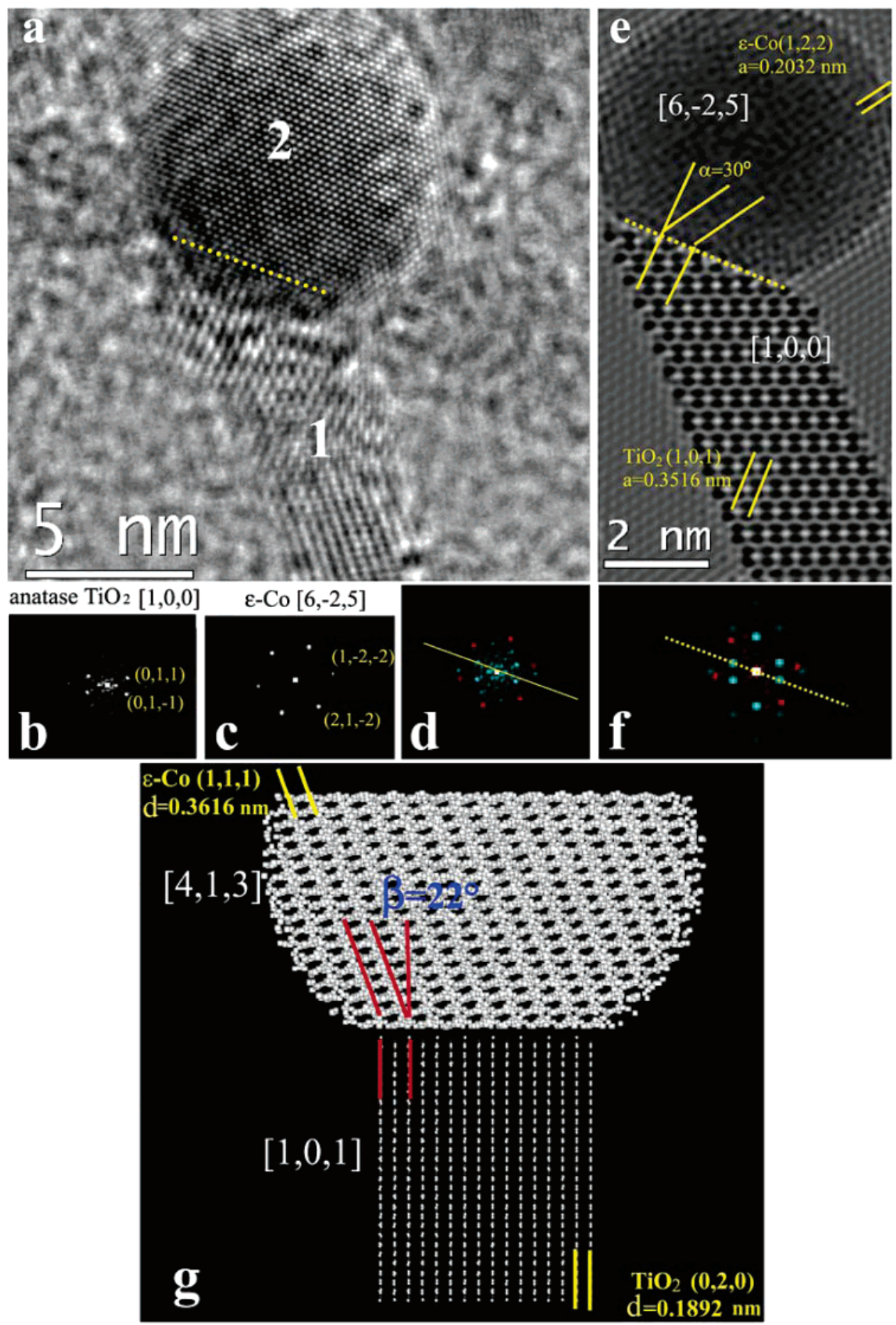

Figure 4. (a) HRTEM image of a single matchstick-shaped HNC. The dotted yellow line indicates the relative orientation of the interfacial plane between the two domains, as inferred from fast Fourier transform (FFT) analyses in panels b-d. (b-c) FFT patterns obtained from the rodlike anatase $\mathrm{TiO}_{2}$ section (marked as " 1 " in panel (a) in its $[1,0,0]$ zone axis and from the spherical $\epsilon$-Co section (marked as " 2 " in panel (a) in its $[6,-2,5]$ zone axis, respectively. (d) Merging of the two FFTs in panels (b-c): blue refers to $\mathrm{TiO}_{2}$, while red relates to $\epsilon-\mathrm{Co}$; the yellow line is consistent with the orientation of the interface. (e) HRTEM simulation for a simplified interface where the two domains are positioned as in (a). The projection $\sigma^{\prime}$ of every 3 adjacent $\{1,2,2\}_{\epsilon-\text { Co }}$ planes on the interface, that is $\sigma^{\prime}=3 \mathrm{~d}_{\{1,2,2\}}^{\mathrm{Co}} / \cos (\alpha)$, matches regularly with the projection $\sigma^{\prime \prime}$ of every 2 consecutive $\{1,0,1\}_{\mathrm{TiO} 2}$ spacings, that is equal to $\sigma^{\prime \prime}=2 \mathrm{~d}_{\{1,0,1\}}^{\mathrm{TiO}_{2}}$. The mismatch on this unidimensional interface can be calculated as: $\left(\sigma^{\prime}-\sigma^{\prime \prime}\right) /\left(\sigma^{\prime \prime}\right) 100=0.1 \%$. (f) Simulated joint FFT pattern relative to panel (e). (g) Atomistic model representing the $\mathrm{HNC}$ in panel (e) after a $90^{\circ}$ rotation with respect to the interfacial plane. Now, $\epsilon$-Co is in its $[4,1,3]$ zone axis, while $\mathrm{TiO}_{2}$ is approximately in its $[1,0,1]$ zone axis. The projection $\rho^{\prime}$ of each $\{1,1,1\}_{\epsilon-\mathrm{Co}}$ on the interface, that is given by $\rho^{\prime}=$ $\mathrm{d}_{\{1,1,1\}}^{\mathrm{Co}} / \cos (\beta)$, coincides regularly to the projection $\rho^{\prime \prime}$ of two consecutive $\{0,2,0\}_{\mathrm{TiO} 2}$ planes, that is given by $\left.\rho^{\prime \prime}=2 \mathrm{~d}_{\{0,2,0\}}^{\mathrm{TiO}}\right\}$. The misfit corresponding to this unidimensional interface can be calculated as: $\left(\rho^{\prime}-\rho^{\prime \prime}\right) /\left(\rho^{\prime \prime}\right) 100=0.26 \%$.

results in a $\sim 5 \%$ misfit. ${ }^{32}$ This is further corroborated by the corresponding HRTEM simulation in panel e. The atomistic model for heterostructure in panel f reveals that, on the unidimensional interface orthogonal to that observed 

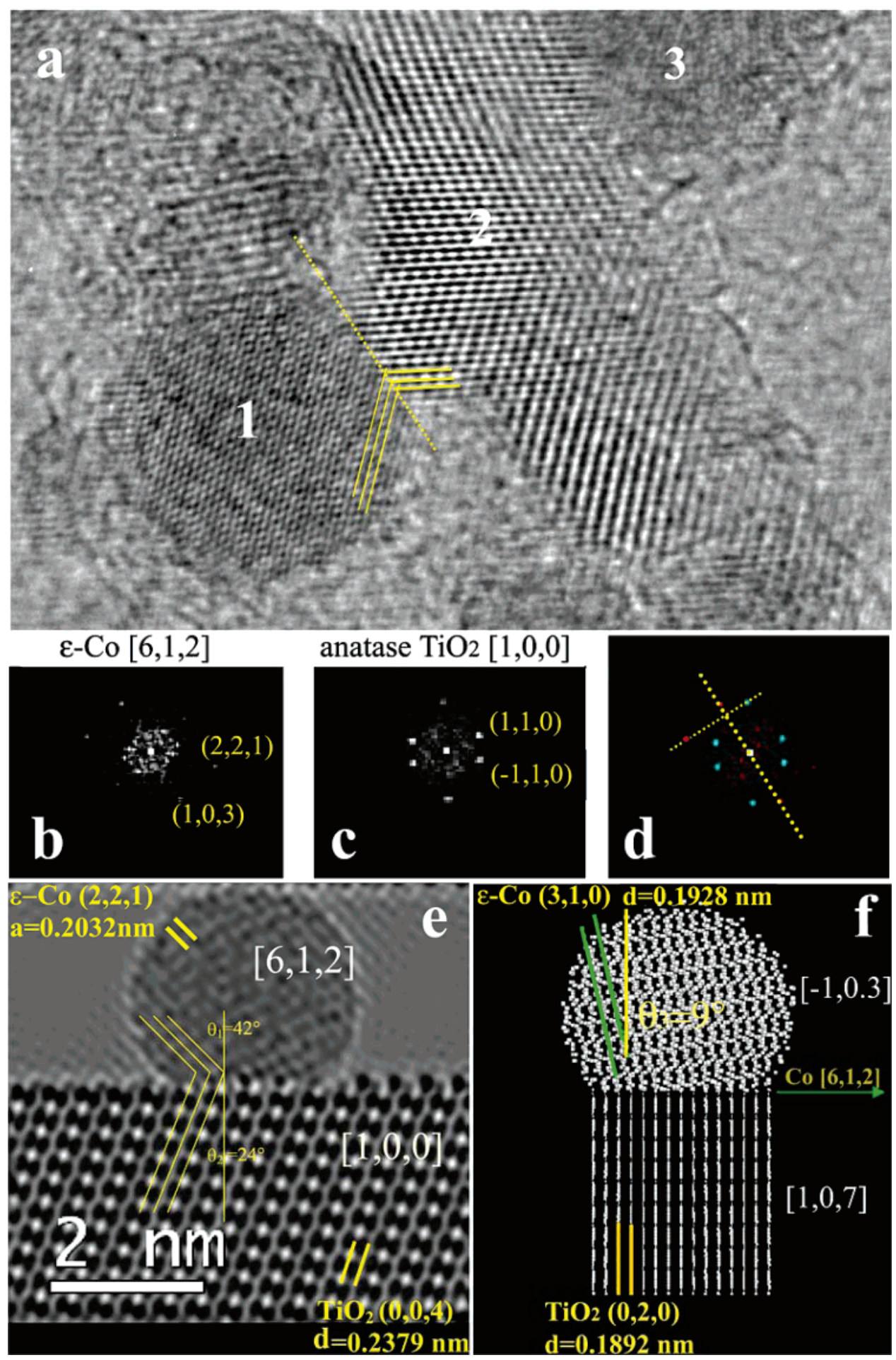

Figure 5. (a) HRTEM image of a portion of a $\mathrm{TiO}_{2}$ nanorod (labeled as "2") carrying multiple $\epsilon$-Co particles (labeled as " 1 " and " 3 ") on its longitudinal sides. The dotted yellow line marks the relative orientation of the interfacial plane between the metal particle " 1 " and the oxide domain (the particle " 3 " is out of focus), as inferred from fast Fourier transform (FFT) analyses in panels (b-d). (b-c) FFT patterns obtained from the $\epsilon$-Co sphere marked as " 1 " in its [6,1,2] zone axis and from the rodlike $\mathrm{TiO}_{2}$ domain in its $[1,0,0]$ zone axis. (d) Merging of the two FFTs in panels $\left(\mathrm{b}-\mathrm{c}\right.$ ): blue refers to $\mathrm{TiO}_{2}$, and red indicates $\epsilon$-Co; the yellow line marks the orientation of the interface. (e) HRTEM simulation for a simplified interface where the two domains are positioned as in panel (a). The projection $\delta^{\prime}$ of each $\{2,2,1\}_{\epsilon-\text { Co }}$ plane on the interface, that is $\delta^{\prime}=\mathrm{d}_{\{2,2,1\}}^{\mathrm{Co}} / \cos \left(\theta_{1}\right)$, coincides regularly with the projection $\delta^{\prime \prime}$ of each $\{0,0,4\}_{\mathrm{TiO} 2}$ plane, that equals $\delta^{\prime \prime}=$ $\mathrm{d}_{\{0,0,4\}}^{\mathrm{TiO}_{2}} / \cos \left(\theta_{2}\right)$. The misfit on this unidimensional interface can be calculated as follows: $\left(\delta^{\prime}-\delta^{\prime \prime}\right) /\left(\delta^{\prime \prime}\right) 100=5 \%$. (f) Atomistic model representing the $\mathrm{HNC}$ in panel (e) after a $90^{\circ}$ rotation with respect to the interfacial plane. Now $\epsilon$-Co is found in its [-1,0,3] zone axis, while $\mathrm{TiO}_{2}$ is approximately in its $[1,0,7]$ zone axis. Under this orientation, the projection $\eta^{\prime}$ of each $\{3,1,0\}_{\epsilon-\mathrm{Co}}$ plane on the interface, that is given by $\eta^{\prime}=\mathrm{d}_{\{3,1,0\}}^{\mathrm{Co}} / \cos \left(\theta_{3}\right)$, matches regularly with the projection $\eta^{\prime \prime}$ of each $\{0,2,0\}_{\mathrm{TiO} 2}$ plane, that equals $\eta^{\prime \prime}=\mathrm{d}_{\{0,2,0\}}^{\mathrm{TiO}}$. The lattice misfit on this unidirectional interface can be calculated as: $\left(\eta^{\prime}-\eta^{\prime \prime}\right) /\left(\eta^{\prime \prime}\right) 100=0.6 \%$ 
Scheme 1. Mechanism for Surfactant-Controlled Co Deposition on $\mathrm{TiO}_{2}$ Nanorod Seeds

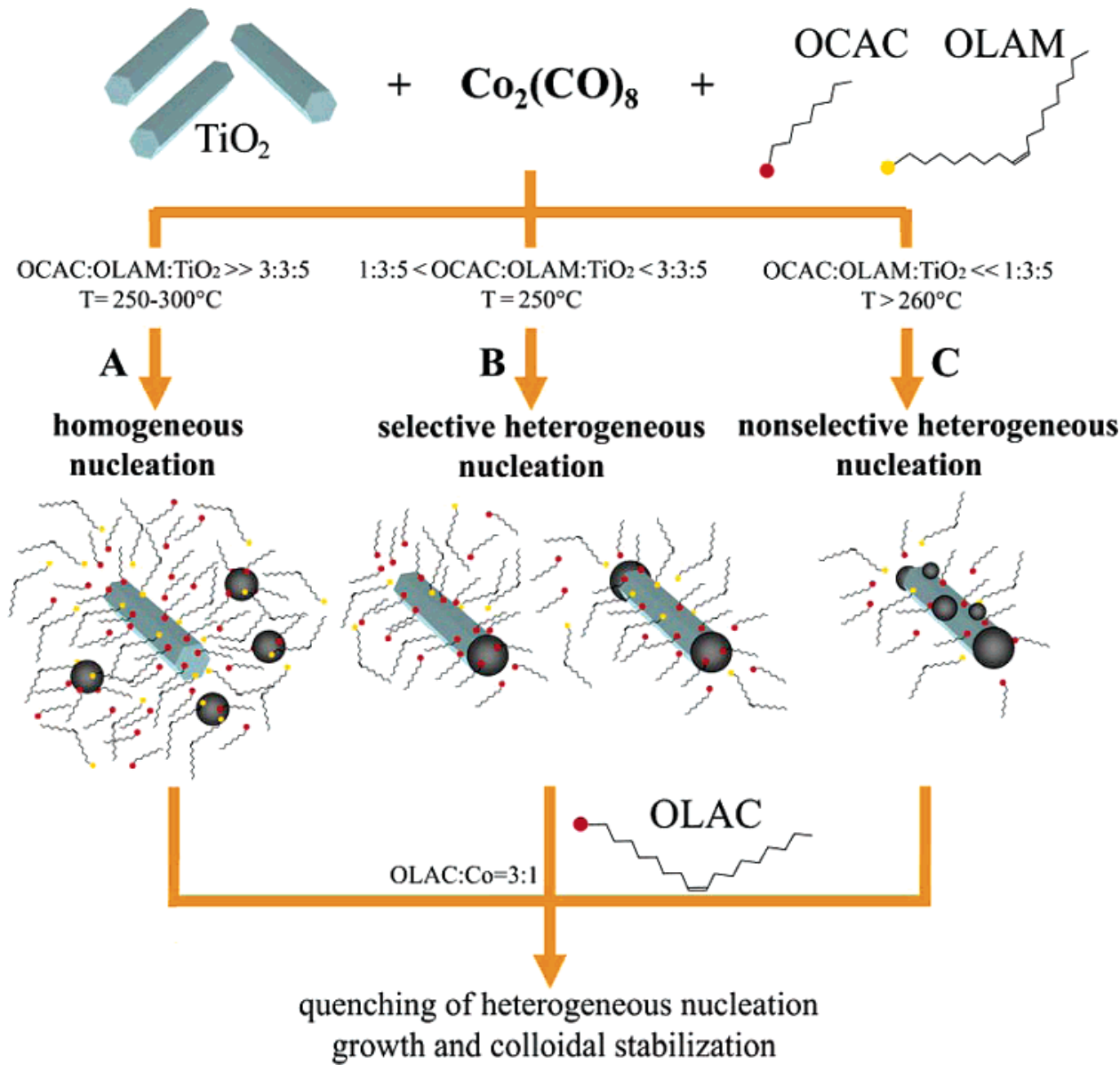

in the experimental image, each $\{3,1,0\}_{\epsilon-\text { Co }}$ plane coincides systematically with a single $\{0,2,0\}_{\mathrm{TiO} 2}$ plane. The associated strain misfit is as low as $0.6 \% .^{32}$

All of the above-reported syntheses experiments indicate that the $\mathrm{HNC}$ are formed through direct deposition of $\mathrm{Co}$ onto the preformed $\mathrm{TiO}_{2} \mathrm{NR}$ seeds, followed by gradual enlargement of the metal domains over time. The final $\mathrm{Co}$ size can be tuned by adjusting the $\mathrm{TiO}_{2} / \mathrm{Co}_{2}(\mathrm{CO})_{8}$ concentration ratio, which regulates the relative balance of cobalt monomer consumption between the nucleation and the growth stages. ${ }^{9,23}$ Other mechanisms were ruled out on the basis of control experiments. ${ }^{29}$

According to the classical nucleation theory (CNT), the activation energy barrier for the growth of an additional material onto pre-existing seeds in a solution (i.e., heterogeneous nucleation) is considerably lower than that for the independent generation of free-standing crystalline embryos (i.e., homogeneous nucleation). ${ }^{33,34}$ Nevertheless, the CNT does not take into account changes in the liquid-solid interface energy induced by adhesion of surfactants to the growing clusters. Our colloidal $\mathrm{TiO}_{2}-\mathrm{Co}$ system can be understood within a simple microscopic mechanism, sketched in Scheme 1, in which the ligand composition and concentration in the solution are the most influential parameters in governing the growth mode of the heterostructures. In this picture, the degree of heterogeneous Co growth and the evolution of the heterostructure topology reflect relevant changes in the surface reactivity of the NRs as a function of the reaction conditions.

A diluted binary surfactant mixture has been selected as a suitable growing medium for the HNCs (note that displacement of the ligands that initially cap the as-prepared NRs provides only a negligible contribution to the total concentration of surfactants typically used in the HNC syntheses). Whereas OLAM preserves the NR solubility without significantly interfering with the metal deposition process, however, carboxylic acids can influence the HNC formation in a critical way. Because of their strong affinity to both $\mathrm{TiO}_{2}{ }^{30}$ and Co surfaces, ${ }^{31}$ these stabilizers are able to build such a compact organic shell around the seeds as to even inhibit their reaction with the available monomers. This reaction regime sets in irrespective of the temperature if the initial acid/ $/ \mathrm{TiO}_{2}$ molar ratio exceeds a limiting value, which is much lower for OLAC than for OCAC, owing to the higher sterical hindrance of the former. Under such circumstances, the cobalt monomers that are slowly supplied to the mixture start to accumulate in the solution, which inevitably leads to crossing of the supersaturation threshold after some time. ${ }^{1,3,29}$ As a consequence, a substantial burst of homogeneous nucleation takes place, yielding highly stabilized Co NCs (Scheme 1, path A). In contrast, the result of triggering $\mathrm{HNC}$ formation already at $250{ }^{\circ} \mathrm{C}$ in the presence of the short-chain OCAC molecules at a reasonably low OCAC/ $\mathrm{TiO}_{2}$ ratio suggests that the $\mathrm{TiO}_{2}$ seeds are chemically 
accessible when they are protected by a permeable organic shell of nonbulky ligands (Scheme 1, paths B-C). In such cases, any excess of monomers can be depleted during heterogeneous growth of the HNCs, thus greatly suppressing the generation of separate Co NCs. Taken together, all of these arguments fully justify our technique of changing the surfactant composition along the synthesis course: the initial presence of OCAC serves to temporally confine heterogeneous Co nucleation to the initial reaction period, while the subsequent massive addition of OLAC quenches any further metal deposition and guarantees aggregation-free completion of HNC growth.

When Co grows on the NRs, it never coats the seeds uniformly, but it segregates in distinct domains without forming any intermediate metal layer. The incomplete Co "wetting" of the $\mathrm{TiO}_{2}$ substrate is reminiscent of the Volmer-Weber type growth of quantum dot islands on mismatched substrates by heteroepitaxial deposition techniques, e.g., molecular beam epitaxy. Within such a context, the change in the total surface energy, $\Delta \gamma$, that accompanies the $\mathrm{Co}$ deposition process on a $\mathrm{TiO}_{2}$ nanorod substrate, should fulfill the condition: ${ }^{35}$

$$
\Delta \gamma=\gamma_{\mathrm{TiO} 2}-\left(\gamma_{\mathrm{Co}}+\gamma_{\mathrm{i}}\right)<0
$$

where $\gamma_{\mathrm{TiO} 2}$ and $\gamma_{\mathrm{Co}}$ are the solution-solid interfacial energies of the respective material domains, which are affected by surfactant adhesion, and $\gamma_{\mathrm{i}}$ is the solid-solid $\mathrm{TiO}_{2} / \mathrm{Co}$ interfacial energy, which is mostly related to the mismatch between the two lattices. In this regard, the synthesis dynamics described in Scheme 1 can be equally understood in terms of energy balance. The formation of HNCs becomes convenient when it is accompanied by a significant decrease in the total surface energy of the system with respect to the case in which only separate $\mathrm{TiO}_{2}$ NRs and Co NCs are formed. The attainment of an inorganic $\mathrm{TiO}_{2}-\mathrm{Co}$ junction can be actually regarded as a means to compensate for the increases in both $\gamma_{\mathrm{TiO} 2}$ and $\gamma_{\mathrm{Co}}$, caused by ineffective surface passivation at low surfactant content. ${ }^{33-35}$ Importantly, the feasibility of switching Co deposition between the tip and the longitudinal sides of seeds demonstrates that the $\mathrm{TiO}_{2}$ NRs possess facet-dependent reactivity. The microscopic origin of such growth mechanism can be deduced from the following arguments.

The two $\mathrm{TiO}_{2}-\mathrm{Co}$ junction structures, which we have more frequently observed, appear to be energetically convenient in terms of interfacial strain..$^{7,8,23,32}$ In contrast to what occurs in highly mismatched $\mathrm{TiO}_{2}$ NR-based heterostructures, ${ }^{23}$ here the coherent attachment between the two materials is unlikely to induce strong lattice distortion fields through the oxide section, which would therefore explain why the seeds can eventually sustain multiple heterogeneous nucleation events. As the maximum lattice misfit values associated with either heterointerface types do not diverge from each other to a great extent, the topologically controlled Co deposition could hardly originate from facet-dependent interfacial strain only. ${ }^{7,8,23}$

Our experimental evidence is that the control over the $\mathrm{HNC}$ topology is achieved by regulation of the sole OCAC/
$\mathrm{TiO}_{2}$ concentration ratio. Therefore, we deduce that the sets of conditions that selectively verify eq 1 for either HNC topologies should be strongly determined by the OCACinduced modulation of both $\gamma_{\mathrm{TiO} 2}$ and $\gamma_{\mathrm{Co}}$, rather than by small differences in $\gamma_{i}$ at the relevant junction points.

At suitable $\mathrm{OCAC} / \mathrm{TiO}_{2}$ ratios, matchstick- and dumbbellshaped HNCs are obtained (Scheme 1, path B), which implies that the basal sides of the NRs are more chemically accessible, as they are less efficiently organic-passivated than other facets. ${ }^{15-18}$ The fact that substantial decrease in the OCAC/TiO 2 ratio promotes Co growth on the NR sidewalls further supports such a hypothesis. Indeed, under such conditions, the ligand coverage on the longitudinal facets of the seeds becomes so poor that Co starts to nucleate also on these locations (Scheme 1, path C). The formation of multiply Co-decorated HNCs reflects further accentuation of this tendency when larger NRs and/or at high temperatures are employed. Indeed, larger seeds offer more extended surfaces available for Co nucleation, while higher temperatures (270$300{ }^{\circ} \mathrm{C}$ ) promote a faster ligand exchange between the NRs and the solution as well as higher monomer diffusion rate, both of which enhance the reactivity of the seeds. ${ }^{1,23}$ It is notable that neither the Co-tipped NRs nor the multiply Codecorated NRs undergo further topological evolution after the Co domains have completed their growth and the monomer concentration has considerably been depleted. Hence, the mechanism for the site-selective Co growth on $\mathrm{TiO}_{2} \mathrm{NRs}$ is clearly different from that leading to $\mathrm{Au}$ deposition on $\mathrm{CdS}$ and CdSe nanorods. ${ }^{17,18}$ For the latter systems, the location and the number of the metal domains, as well as their size, evolve simultaneously over time due to chemical activation of nanorod surface defects that act as nucleation centers and to electrochemical metal particle ripening, respectively. ${ }^{17,18}$

Preliminary magnetic measurements on the HNCs are presented in Figure 6. In panel a, the hysteresis loops, measured at $300 \mathrm{~K}$ for two HNC samples with mean Co domain size of $3.7 \pm 0.7 \mathrm{~nm}$ and $5.2 \pm 0.8 \mathrm{~nm}$, are compared with that of $6.5 \pm 0.7 \mathrm{~nm}$ Co NCs. Note that the diamagnetic contribution of the $\mathrm{TiO}_{2}$ component, measured independently, is negligible. All the curves evidence neither remanence nor coercivity, indicating a superparamagnetic behavior. The saturation magnetization values, $M_{\mathrm{S}}$, per gram of cobalt, are progressively reduced as the metal domains become smaller regardless of the $\mathrm{TiO}_{2}$ presence, which is consistent with the size-dependent behavior of $\epsilon$-Co in this dimensional regime. ${ }^{31}$ Accordingly, panel $\mathrm{b}$ shows coercive fields ranging from 500 to $850 \mathrm{Oe}$ at $5 \mathrm{~K}$. As an indirect confirmation, the magnetic properties the HNC powders after prolonged exposure to air are found to be severely modified due to the oxidation of $\epsilon$-Co to antiferromagnetic $\mathrm{CoO}^{29}$ In panel c, the temperature dependence of the magnetization is fully consistent with the superparamagnetic behavior detected at $300 \mathrm{~K}$. Interestingly, the average blocking temperature, $T_{\mathrm{B}}$ (at which the respective curves reach their maximum), relative to the heterostructure samples, lies in between 10$60 \mathrm{~K}$, which falls below from the value of $130 \mathrm{~K}$ estimated for the bare Co NCs. Moreover, the analyses of HNC samples 

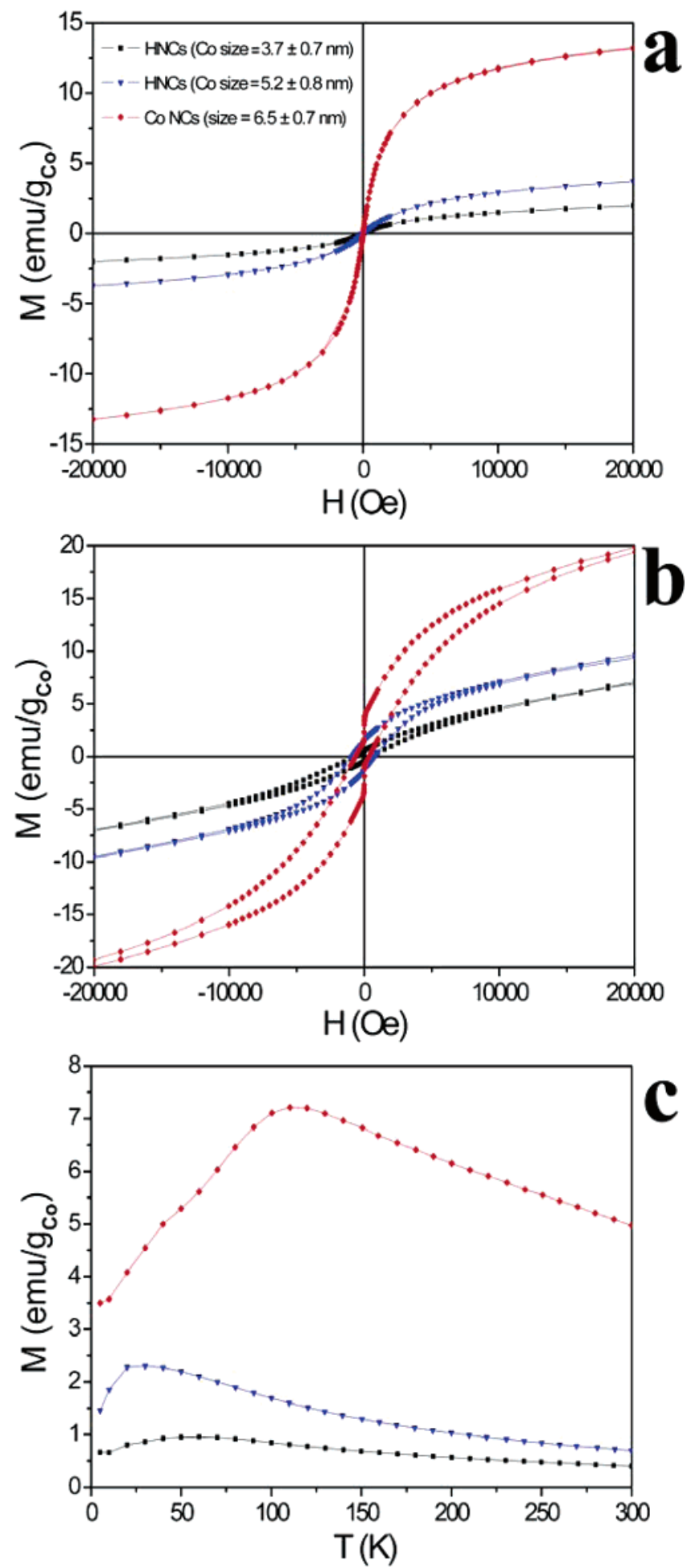

Figure 6. Magnetic measurements on HNC samples: $(a-b)$ Hysteresis loops at 300 and at $5 \mathrm{~K}$, respectively, (c) thermal evolution of the magnetization under an applied field of $1 \mathrm{kOe}$.

with intermediate Co size evidence no clear dependence of $T_{\mathrm{B}}$ on the dimensions of the metal domains. These results can be not interpreted straightforwardly by the Néel model, which in fact predicts $T_{\mathrm{B}}$ to scale up linearly with the NC magnetic volume, $V_{\mathrm{NC}}$ :

$$
T_{\mathrm{B}}=K_{\mathrm{A}} \cdot V_{\mathrm{NC}} / 25 K_{\mathrm{B}}
$$

where $K_{\mathrm{B}}$ is the Boltzman constant, and the anisotropy constant $K_{\mathrm{A}}$ comprises several contributions. ${ }^{36,37}$ One possible explanation could be that, as the two materials communicate through a rather extended inorganic junction, proximity effects at the interface can unusually influence the magnetic anisotropy term. ${ }^{9}{ }^{11,13}$ Further experiments are in progress to understand the origin of such phenomena.

In conclusion, we have described a colloidal seeded approach to synthesize a new type of hybrid nanocrystal based on Co-decorated $\mathrm{TiO}_{2}$ nanorods, whose topology is controlled by a facet-selective surfactant adhesion mechanism. The analyses of the epitaxial relationships between the two domains as function of the synthesis conditions extends the basic knowledge on the interplay between surface energy and interfacial strain during the formation of a nanocrystal heterostructure. Our work suggests that refined control of the nanocrystal surface chemistry by selection of suitable ligands could lead to a more rational design of heterostructures, facilitating access to other combinations of nanomaterials.

The present $\mathrm{TiO}_{2}-\mathrm{Co}$ HNCs could be interestingly exploited as bifunctional catalysts ${ }^{25,26}$ (for instance, in the Fischer-Tropsch synthesis of hydrocarbons), ${ }^{25 \mathrm{~d}-\mathrm{h}}$ in which the dispersion of the metal and oxide components is finely arranged at the nanoscale. Because of the intimate magnetic metal/semiconductor oxide connection, such HNCs could additionally work as magnetically recoverable photocatalysts. This association of materials would improve the photocatalytic performances of $\mathrm{TiO}_{2}$ through metal-enhanced charge carrier delocalization, ${ }^{26}$ simultaneously enabling fluidization and local heating production in the solution upon application of an external magnetic field. Finally, these heterostructures could serve as convenient systems to study how the properties of a technologically important magnetic metal are affected by the overlapping of its electronic structure with that of an either unexcited or photoexcited semiconductor, which may lead to novel magneto-optical applications.

Acknowledgment. This work was partially supported by the European project SA-NANO (contract no. STREP 013698) and by the Italian Ministry of Research (contracts no. RBLA03ER38 and no. RBIN048TSE). We thank Roberto Lassandro and Dr. Giuseppe Ciccarella for technical assistance in XRD measurements and ICP analyses, respectively.

Supporting Information Available: Details on the HNC synthesis; daylight pictures demonstrating magnetically induced separation of HNCs; control experiments; TEM images of Co $\mathrm{NCs}$ prepared without $\mathrm{TiO}_{2}$ and of $\mathrm{HNCs}$ at different surfactant concentration; experimental details on ICP, TEM, HRTEM, STEM-HAADF, XRD, and magnetic measurements; XRD patterns and magnetic measurements of air-oxidized samples. This material is available free of charge via the Internet at http://pubs.acs.org.

\section{References}

(1) (a) Burda, C.; Chen, X. B.; Narayanan, R.; El-Sayed, M. A. Chem. Rev. 2005, 105, 1025. (b) Jun, Y.-w.; Choi, J.-s. C.; Cheon, J. Angew. Chem., Int. Ed. 2006, 45, 3414. 
(2) (a) Pellegrino, T.; Kudera, S.; Liedl, T.; Munoz Javier, A.; Manna, L.; Parak, W. J. Small 2005, 1, 48. (b) Ma, D. L.; Guan, J. W.; Normandin, F.; Denommee, S.; Enright, G.; Veres, T.; Simard, B. Chem. Mater. 2006, 18, 1920. (c) Huh, Y. M.; Jun, Y. W.; Song, H. T.; Kim, S.; Choi, J. S.; Lee, J. H.; Yoon, S.; Kim, K. S.; Shin, J. S.; Suh, J. S.; Cheon, J. J. Am. Chem. Soc. 2005, 127, 12387. (d) Lee, J.-H.; Jun, Y.-w.; Yeon, S.-I.; Shin, J.-S.; Cheon, J. Angew. Chem., Int. Ed. 2006, 45, 8160.

(3) Cozzoli, P. D.; Pellegrino, T.; Manna, L. Chem. Soc. Rev. 2006, 35 , 1195.

(4) Teranishi, T.; Inoue, Y.; Nakaya, M.; Oumi, Y.; Sano, T. J. Am. Chem. Soc. 2004, 126, 9914.

(5) Choi, S. H.; Kim, E. G.; Hyeon, T. J. Am. Chem. Soc. 2006, 128, 2520.

(6) Gu, H. W.; Zheng, R. K.; Zhang, X. X.; Xu, B. J. Am. Chem. Soc. 2004, 126, 5664

(7) Kwon, K. W.; Shim, M. J. Am. Chem. Soc. 2005, 127, 10269.

(8) Kwon, K.-W. L. B. H.; Shim, M. Chem. Mater. 2006, 18, 6357.

(9) Pellegrino, T.; Fiore, A.; Carlino, E.; Giannini, C.; Cozzoli, P. D.; Ciccarella, G.; Respaud, M.; Palmirotta, L.; Cingolani, R.; Manna, L. J. Am. Chem. Soc. 2006, 128, 6690.

(10) Gao, X.; Yu, L.; MacCuspie, R.; Matsui, H. Adv. Mater. 2005, 17, 426.

(11) Yu, H.; Chen, M.; Rice, P. M.; Wang, S. X.; White, R. L.; Sun, S. H. Nano Lett. 2005, 5, 379.

(12) Gu, H. W.; Yang, Z. M.; Gao, J. H.; Chang, C. K.; Xu, B. J. Am. Chem. Soc. 2005, 127, 34

(13) Shi, W.; Zeng, H.; Sahoo, Y.; Ohulchanskyy, T. Y.; Ding, Y.; Wang, Z. L.; Prasad, P. N. Nano Lett. 2006, 6, 875.

(14) Teranishi, T.; Saruyama, M.; Nakaya, M.; Kanehara, M. Angew. Chem., Int. Ed. 2007, 46, 1713.

(15) Milliron, D. J.; Hughes, S. M.; Cui, Y.; Manna, L.; Li, J. B.; Wang, L. W.; Alivisatos, A. P. Nature 2004, 430, 190.

(16) Mokari, T.; Rothenberg, E.; Popov, I.; Costi, R.; Banin, U. Science 2004, 304, 1787.

(17) Mokari, T.; Sztrum, C. G.; Salant, A.; Rabani, E.; Banin, U. Nat. Mater. 2005, 4, 855.

(18) Saunders, A. E. P. I.; Banin, U. J. Phys. Chem. B 2006, 110, 25421.

(19) Kudera, S.; Carbone, L.; Casula, M. F.; Cingolani, R.; Falqui, A.; Snoeck, E.; Parak, W. J.; Manna, L. Nano Lett. 2005, 5, 445.

(20) Carbone, L.; Kudera, S.; Giannini, C.; Ciccarella, G.; Cingolani, R.; Cozzoli, P. D.; Manna, L. J. Mater. Chem. 2006, 16, 3952.

(21) Yang, J.; Elim, I. H.; Zhang, Q. B.; Lee, J. Y.; Ji, W. J. J. Am. Chem. Soc 2006, 128, 11921.

(22) Pacholski, C.; Kornowski, A.; Weller, H. Angew. Chem., Int. Ed. 2004, 43, 4774 .

(23) Buonsanti, R.; Grillo, V.; Carlino, E.; Giannini, C.; Curri, M. L.; Innocenti, C.; Sangregorio, C.; Achterhold, K.; Parak, F. G.; Agostiano, A.; Cozzoli, P. D. J. Am. Chem. Soc 2006, 128, 16953.

(24) Salant, A. A.-S. E.; Banin, U. J. Am. Chem. Soc 2006, 128, 10006.

(25) (a) Benjaram, M.; Reddy, A. K. Catal. Rev. 2005, 47, 257. (b) Comotti, M. L.; W.-C.; Spliethoff, B.; Schuth, F. J. Am. Chem. Soc
2006, 128, 917-924. (c) Borodziński, A.; Bond, G. C. Catal. Rev 2006, 48, 91. (d) Van Der Laan, G. P.; Beenackers A. A. C. M. Catal. Rev. 1999, 41, 255. (e) Dry, M. E. J. Chem. Technol. Biotechnol. 2002, 77, 43. (f) Suriye, K. P. P.; Jongsomjit, B. Ind. Eng. Chem. Res. 2005, 44, 6599. (g) Linghu, W.; Liu, X.; Li, X.; Fujimoto, K. Catal. Lett. 2006, 108, 11. (h) Elbashir, N. O.; Dutta, P.; Manivannan, A.; Seehra, M. S.; Roberts, C. B. Appl. Catal. A 2005, 285, 169.

(26) (a) Subramanian, V.; Wolf, E. E.; Kamat, P. V. J. Am. Chem. Soc. 2004, 126, 4943. (b) Maeda, K.; Teramuda, K.; Lu, D.; Saito, N.; Inoue, Y.; Domen, K. Angew. Chem., Int. Ed. 2006, 118, 7970. (c) Wood, A.; Giersig, M.; Mulvaney, P. J. Phys. Chem. B 2001, 105, 8810. (d) Cozzoli, P. D.; Fanizza, E.; Comparelli, R.; Curri, M. L.; Agostiano, A.; Laub, D. J. Phys. Chem. B 2004, 108, 9623.

(27) (a) Wallace, W. T.; Min, B. K.; Goodman, D. W. Top. Catal. 2005, 34, 17. (b) Bourikas, K.; Kordulis, C.; Lycourghiotis, A. Catal. Rev. 2006, 48, 363. (c) Zheng, N.; Stucky, G. D. J. Am. Chem. Soc 2006, 128, 14278. (d) Yan, W. M. S. M.; Pan, Z.; Overbury, S. H.; Dai, S. J. Am. Chem. Soc. 2005, 127, 10480. (e) Cozzoli, P. D.; Comparelli, R.; Fanizza, E.; Curri, M. L.; Agostiano, A.; Laub, D. J. Am. Chem. Soc. 2004, 126, 3868. (f) Cozzoli, P. D.; Curri, M. L.; Giannini, C.; Agostiano, A. Small 2006, 2, 413.

(28) (a) Li, J.; Zeng, H. C. Angew. Chem., Int. Ed. 2005, 44, 4342. (b) Du, J. Z. J.; Liu, Z.; Han, B.; Jiang, T.; Huang, Y. Langmuir 2006, 22, 1307. (c) Tom, R. T.; Nair, A. S.; Singh, N.; Aslam, M.; Nagendra, C. L.; Philip, R.; Vijayamohanan, K.; Pradeep, T. Langmuir 2003, 19, 3439.

(29) See Supporting Information.

(30) (a) Cozzoli, P. D.; Kornowski, A.; Weller, H. J. Am. Chem. Soc. 2003, 125, 14539. (b) Seo, J. W.; Jun, Y. W.; Ko, S. J.; Cheon, J. J. Phys. Chem. B 2005, 109, 5389.

(31) (a) Puntes, V. F.; Krishnan, K. M.; Alivisatos, A. P. Science 2001, 291, 2115. (b) Park, J.-I.; Kang, N.-J.; Jun, Y.-W.; Oh, S. J.; Ri, H.-C.; Cheon, J. Chem. Phys. Chem. 2002, 3, 543.

(32) Randle, V. The Role of the Coincidence Site Lattice in Grain Boundary Engineering; Woodhead Publishing Limited: Cambridge, U.K., 1997.

(33) Mullin, J. W. Crystallization, 3rd ed; Butterworth-Heinemann: Oxford, 1997.

(34) Sugimoto, T. Monodisperse Particles; Elsevier: Amsterdam, 2001.

(35) Markov, I. V. Crystal Growth for Beginners: Fundamentals of Nucleation, Crystal Growth, and Epitaxy; World Scientific: Singapore, 2003.

(36) Surface Effects in Magnetic Nanoparticles; Fiorani, D., Ed.; Springer: New York, 2005.

(37) Dormann, J. L.; Fiorani, D.; Tronc, E. Magnetic Relaxation in FineParticle Systems. In Advances in Chemical Physics; Prigogine, I., Rice, A., Eds.; John Wiley \& Sons: New York, 1997; Vol. 98.

\section{NL070550W}

